\title{
Development of Optical-fiber-based Neutron Detector Using Li glass Scintillator for an Intense Neutron Field
}

\author{
Akihisa Ishikawa, ${ }^{1 *}$ Atsushi Yamazaki,${ }^{1}$ Kenichi Watanabe,${ }^{1}$ \\ Sachiko Yoshihashi, ${ }^{1}$ Akira Uritani, ${ }^{1}$ Yoshinori Sakurai, ${ }^{2}$ \\ Hiroki Tanaka, ${ }^{2}$ Ryo Ogawara, ${ }^{3}$ Mitsuru Suda,${ }^{3}$ and Tsuyoshi Hamano ${ }^{3}$ \\ ${ }^{1}$ Department of Applied Energy Engineering, Nagoya University, Furo-cho, \\ Chikusa-ku, Nagoya 464-8603, Japan \\ ${ }^{2}$ Institute for Integrated Radiation and Nuclear Science, Kyoto University, 2, \\ Asashiro-Nishi, Kumatori-cho, Sennan-gun, Osaka 590-0494, Japan \\ ${ }^{3}$ National Institutes for Quantum and Radiological Science and Technology, \\ 4-9-1 Anagawa, Inage-ku, Chiba-shi, Chiba 263-8555, Japan
}

(Received December 19, 2019; accepted March 19, 2020)

Keywords: optical-fiber-based neutron detector, real-time measurement, Li glass, Eu:LiCaAlF $\mathrm{LiF} / \mathrm{Eu}: \mathrm{CaF}_{2}$

The development of a neutron detector for real-time measurements in an intense neutron field is desired. We are developing a quartz optical-fiber-based neutron detector as a real-time neutron monitor. Thus far, Eu:LiCaAlF 6 crystals and $\mathrm{LiF} / \mathrm{Eu}: \mathrm{CaF}_{2}$ eutectics have been used as the scintillators of the quartz optical-fiber-based neutron detectors. These scintillators have high light yields, but relatively long decay time constants, which are the limiting factors for the dynamic range of optical-fiber-based neutron detectors. In this work, we applied a small Li glass scintillator to a quartz optical-fiber-based neutron detector using an optical fiber with high numerical aperture and a UV-curable resin with high transmittance. The fabricated detector showed a clear peak corresponding to neutron events in the pulse height distribution. The radiation resistance was evaluated and no deterioration was observed up to $2 \times 10^{12} \mathrm{n} / \mathrm{cm}^{2}$. The detector output linearity was confirmed up to $450 \mathrm{kcps}$, which was about ten times larger than that of the detector using the conventional Eu: $\mathrm{LiCaAlF}_{6}$ or $\mathrm{LiF} / \mathrm{Eu}: \mathrm{CaF}_{2}$ scintillator.

\section{Introduction}

Neutrons are widely used in various fields, such as homeland security, material science, and medical applications. ${ }^{(1-3)}$ The neutron intensity or neutron flux is one of the most fundamental characteristics examined in neutron experiments. We are developing a quartz optical-fiber-

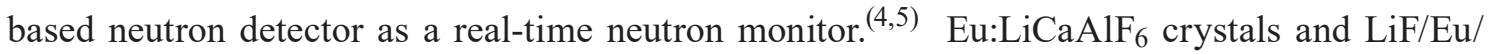
$\mathrm{CaF}_{2}$ eutectics were previously used as neutron scintillators in the quartz optical-fiber-based neutron detector. ${ }^{(4-7)}$ These detectors can show a peak corresponding to neutron events in the pulse height spectrum because these scintillators are transparent and relatively bright. This feature is useful for determining the lower-level discrimination for neutron detection and to

*Corresponding author: e-mail: ishikawa.akihisa@f.mbox.nagoya-u.ac.jp

https://doi.org/10.18494/SAM.2020.2748 
check the signal gain. However, in the configuration of light collection using an optical fiber, it is difficult to show a neutron peak because of its low light collection efficiency. To show a neutron peak in a neutron scintillation detector, uniform light collection from the scintillator and a sufficient number of scintillation photons to be detected by a photodetector are required. In addition, we are applying a quartz optical fiber owing to its high radiation hardness, which has a low numerical aperture (NA) corresponding to low light collection efficiency. To realize a quartz-fiber-based neutron detector showing a peak, it is important to apply a bright scintillator.

Another important feature of the neutron detector is its gamma-ray discrimination capability. For this purpose, we adopt a small neutron scintillator with a diameter of about 200-400 $\mu \mathrm{m}$. Since fast electrons induced in gamma-ray events have ranges long enough to escape from a scintillator before depositing their whole energy, gamma-ray signals have smaller pulse height than neutron ones. For example, $1 \mathrm{MeV}$ electrons, which severely interfere with neutron events, have a range of approximately $2 \mathrm{~mm}$. On the other hand, tritons and particles induced by ${ }^{6} \mathrm{Li}(\mathrm{n}, \alpha)^{3} \mathrm{H}$ reactions have ranges of about 30 and $6 \mu \mathrm{m}$, respectively. This allows the easy discrimination of neutron events from gamma-ray ones. In addition, since the detector using a small scintillator has low sensitivity, it can show excellent linearity between neutron intensity and detected counts even in an intense neutron field. This means that a measurable neutron flux range can be selected by controlling the scintillator size. ${ }^{(5)}$

Although Eu:LiCaAlF 6 crystals and $\mathrm{LiF} / \mathrm{Eu}: \mathrm{CaF}_{2}$ eutectics have high light yields, their long decay time constants (ms order) limit the dynamic range or the maximum counting rate of the detector. In this work, to extend the dynamic range of the optical-fiber-based neutron detector, we applied a lithium glass scintillator to the optical-fiber-based neutron detector. The Li glass scintillator has a relatively short decay time constant of $75 \mathrm{~ns} .{ }^{(8)}$ However, the light yield is 6000 photons/neutron and much lower than the previous ones, such as $\mathrm{Eu}_{\mathrm{LiCaAlF}}$ and $\mathrm{LiF} /$ $\mathrm{Eu}: \mathrm{CaF}_{2}$, which have 40000 and 10000 photons/neutrons, respectively. Matsumoto et al. developed an optical-fiber-based neutron detector using a Li glass scintillator, which can show a neutron peak structure in the pulse height spectrum. ${ }^{(9)}$ Since this detector used a plastic optical fiber, which generally has a relatively high NA, its radiation hardness was insufficient and it was not suitable for measurements in an intense neutron field. In a quartz optical-fiber-based neutron detector using the Li glass scintillator, the number of scintillation photons to be detected at a photodetector is expected to be lower than the previous ones. However, it can be kept at a high level by using a quartz optical fiber with high NA, which has been commercially available recently, and a UV-curable resin with high transmittance at the emission wavelength of the Li glass scintillator. We experimentally evaluated some performance characteristics of the fabricated detector, such as the pulse height spectrum, output linearity, and radiation hardness.

\section{Materials and Methods}

\subsection{Optical-fiber-based neutron detector using a Li glass scintillator}

The structure of the optical-fiber-based neutron detector is shown in Fig. 1. The core diameter and NA of the quartz optical fiber (Thorlabs, FP600URT) were $600 \mu \mathrm{m}$ and 0.5 , 


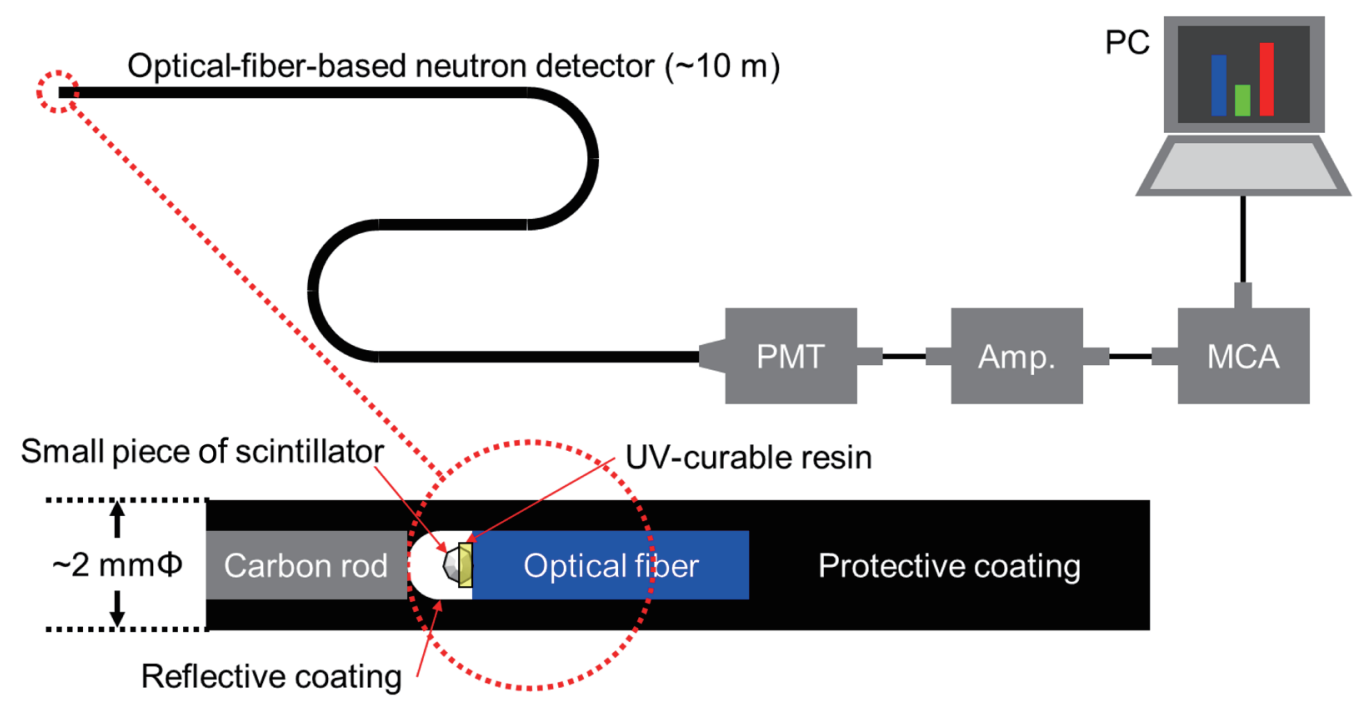

Fig. 1. (Color online) Structure of quartz optical-fiber-based neutron detector. An example of a measurement system is also described.

respectively. The scintillator diameter was controlled to be about 200-400 $\mu \mathrm{m}$. A UV-curable resin (Norland, NOA63) was used to fix a small piece of the Li glass scintillator on the tip of the optical fiber. The transmittance of the resin was nearly $100 \%$ at the emission wavelength of the scintillator. The scintillator fixed at the tip of the optical fiber was coated with a diffusive reflector, which was $\mathrm{TiO}_{2}$ powder. Then, the detector head was shielded from ambient light by using a black heat shrink tube. Figure 1 also shows the whole system of the optical-fiberbased neutron detector including an electrical circuit. The optical fiber was connected to a photomultiplier tube (PMT, Hamamatsu, R9880U-210). The anode signal of the PMT was directly fed into a digital multichannel analyzer (digital MCA, ANSeeN, HSMCA 4414-L-NW).

A photodetector and electrical circuits can be placed at $10 \mathrm{~m}$ from the detector head by using the optical fiber to prevent the exposure of the system to radiation. The optical-fiberbased neutron detector is so small that the detector can be used in a narrow space with little perturbation to measured fields. By controlling the scintillator size, we can selectively suppress the pulse height of $\gamma$-ray events. ${ }^{(4)}$ Therefore, only neutron events can easily be distinguished by a pulse height discrimination technique. Because of these advantages, the optical-fiberbased neutron detector can effectively be used as a real-time neutron monitor in a high-intensity neutron field.

\subsection{Experimental setup}

\subsubsection{Observation of pulse height spectra}

To evaluate the detector response, we directly irradiated the fabricated detector with thermal neutrons at the thermal neutron port E3, in which a neutron guide tube with curvature was 
used, in the Kyoto University Reactor (KUR) in the Institute for Integrated Radiation and Nuclear Science of Kyoto University, Japan. The neutron flux of the E3 port was evaluated to be $4 \times 10^{5} \mathrm{n} / \mathrm{cm}^{2} / \mathrm{s}$ at the reactor power of $1 \mathrm{MW}$ by a gold foil activation method. In this experiment, the signal pulse height spectrum obtained from the fabricated detector was evaluated.

\subsubsection{Radiation hardness test}

The radiation hardness of the fabricated detector was tested at the Heavy Water Neutron Irradiation Facility (HWNIF) in KUR. The irradiation mode was of the mixed type, in which thermal and epithermal neutrons were irradiated. The fabricated detector was surrounded with a polyethylene moderator to increase the thermal neutron flux at the detector head. The thermal neutron flux was evaluated to be $4 \times 10^{8} \mathrm{n} / \mathrm{cm}^{2} / \mathrm{s}$ by the gold foil activation method. A change in the peak position in the pulse height spectrum was monitored during irradiation. Owing to radiation-induced damage, the transmittances of the optical fiber and the UV-curable resin and the reflectance of the reflective coating might be deteriorated. Deterioration of the detector components causes the signal pulse height to decrease.

\subsubsection{Evaluation of detector output linearity}

The detector output linearity was evaluated at the Neutron exposure Accelerator System for Biological Effect Experiments (NASBEE) in the National Institutes for Quantum and Radiological Science and Technology, Japan. The fabricated detector was placed in a polyethylene moderator to enhance the thermal neutron flux at the detector head. Neutrons were generated by ${ }^{9} \mathrm{Be}(\mathrm{p}, \mathrm{n})$ reactions. The proton acceleration energy was $4 \mathrm{MeV}$. The maximum proton beam current was $600 \mu \mathrm{A}$ and the neutron intensity can be changed by adjusting the proton current. When the proton beam current was $600 \mu \mathrm{A}$, the obtained thermal neutron flux was $4 \times 10^{8} \mathrm{n} / \mathrm{cm}^{2} / \mathrm{s}$ at the detector position. The detector counting rate was recorded while increasing the proton beam current. The thermal neutron flux at the detector position was determined by the gold foil activation method.

\section{Results and Considerations}

\subsection{Pulse height spectra}

The pulse height spectra obtained from the fabricated detector are shown in Fig. 2. A clear peak corresponding to neutron events can be confirmed. The capability to create a neutron peak in the pulse height spectrum is useful for determining the lower-level discrimination of the neutron events against the gamma-ray events and background noises. In addition, it is also useful to check the signal gain of the detector. The background noise was measured by shutting out the thermal neutron beam by placing a LiF neutron shielding plate in front of the detector. Although the source of the background noise has not been identified, the background signals 


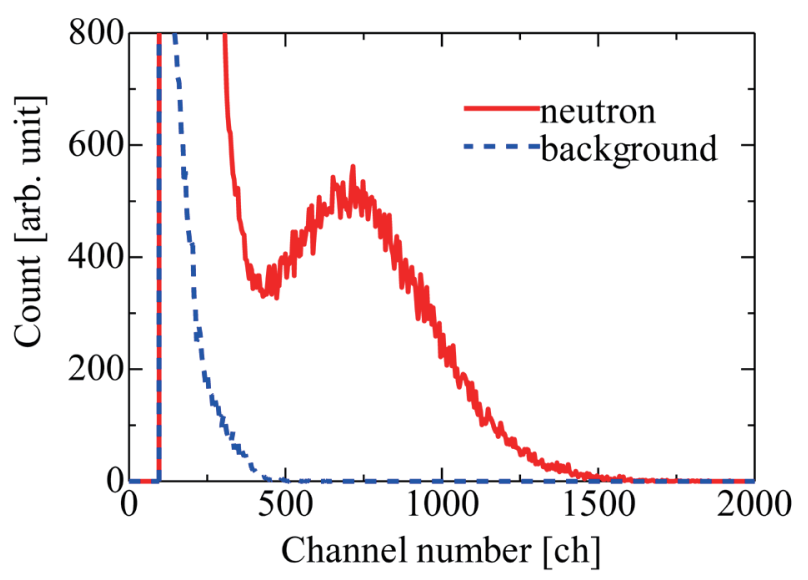

Fig. 2. (Color online) Channel number of neutron peak in pulse height spectrum as function of thermal neutron fluence.

were considered to be attributed to gamma-ray signals and/or electrical noise. The maximum pulse height of the background events was small enough to discriminate them from neutron events. Although the light yield of the Li glass scintillator is smaller than that of the previous Eu: $\mathrm{LiCaAlF}_{6}$ and $\mathrm{LiF} / \mathrm{Eu}: \mathrm{CaF}_{2}$, we can successfully obtain a neutron peak and discriminate gamma-ray events in the pulse height spectrum.

\subsection{Radiation hardness}

Figure 3 shows the channel number of the neutron peak in the pulse height spectrum as a function of thermal neutron fluence. The fabricated detector was irradiated with neutrons up to the thermal neutron fluence of more than $2 \times 10^{12} \mathrm{n} / \mathrm{cm}^{2}$. In this measurement, the channel number of the neutron peak was constant; thus, no deterioration was observed. Even when the deterioration is not negligible at a much higher neutron fluence, the detector sensitivity can be stabilized by adjusting the lower-level discrimination based on autosearching the neutron peak during the measurement.

\subsection{Detector output linearity}

A wide dynamic range is also required in a real-time neutron monitor for an intense neutron field. A sufficiently wide dynamic range enables a single detector to measure neutrons from low to high neutron flux without saturation. Figure 4 shows the detector output linearity obtained from the fabricated detector using a Li glass scintillator. For comparison, the output linearity of the detector using the $\mathrm{LiF} / \mathrm{Eu}: \mathrm{CaF}_{2}$ eutectic scintillator, which was fabricated in the same manner, is also plotted. Although the response of the detector using the $\mathrm{LiF} / \mathrm{Eu}: \mathrm{CaF}_{2}$ eutectic was already saturated at the counting rate of less than $50 \mathrm{kcps}$, the detector using the Li glass scintillator shows no saturation at the counting rate of nearly $450 \mathrm{kcps}$. The dynamic range was extended by using a fast Li glass scintillator. 


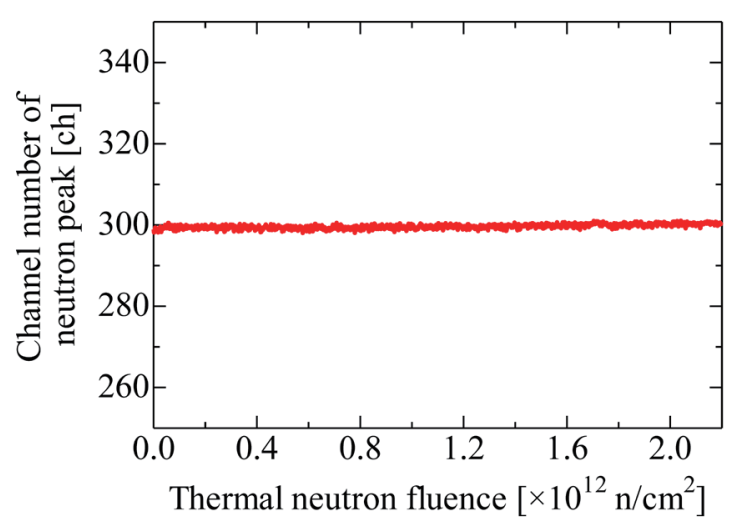

Fig. 3. (Color online) Pulse height spectra of quartz optical-fiber-based neutron detector using Li glass scintillator.

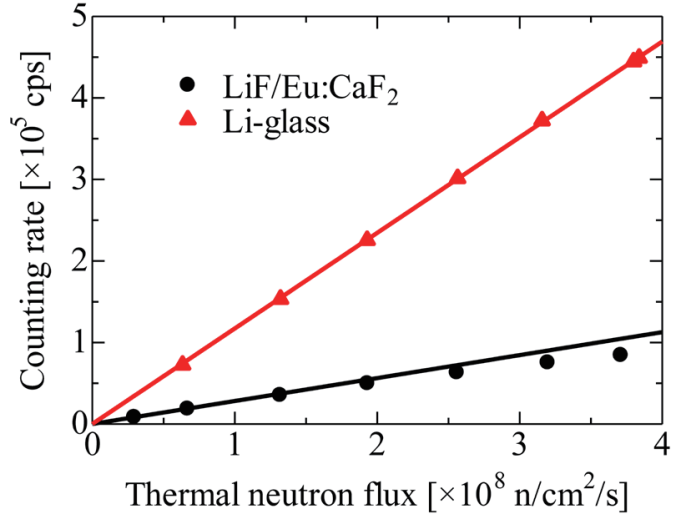

Fig. 4. (Color online) Detector output linearity of fabricated detector using Li glass. That of the detector using $\mathrm{LiF} / \mathrm{Eu}: \mathrm{CaF}_{2}$ eutectic is also plotted for comparison.

\section{Conclusions}

The Li glass scintillator was applied to the quartz optical-fiber-based neutron detector. The quartz optical-fiber-based neutron detector using a Li glass scintillator was fabricated by using a quartz optical fiber with a large NA and a low attenuation, and a UV-curable resin with high transmittance. In the pulse height spectrum of the fabricated detector, a neutron peak was clearly observed. The radiation hardness of the fabricated detector was evaluated, and no deterioration was observed up to the thermal neutron fluence of more than $2 \times 10^{12} \mathrm{n} / \mathrm{cm}^{2}$. The detector output linearity was markedly improved by using the Li glass scintillator. No saturation was confirmed even at the counting rate of nearly $450 \mathrm{kcps}$. These results indicate the effectiveness of the quartz optical-fiber-based neutron detector using a Li glass scintillator as a real-time neutron monitor in an intense neutron field.

\section{Acknowledgments}

This work was partially supported by JSPS KAKENHI Grant Number JP18H03468. This study was partially supported by the Cooperative Research Project of the Research Institute of Electronics, Shizuoka University.

\section{References}

1 R. T. Kouzes, J. H. Ely, L. E. Erikson, W. J. Kernan, A. T. Lintereur, E. R. Siciliano, D. L. Stephens, D. C. Stromswold, R. M. Van Ginhoven, and M. L. Woodring: Nucl. Instrum. Methods Phys. Res., Sect. A 623 (2010) 1035. https://doi.org/10.1016/j.nima.2010.08.021

2 K. Sakasai, S. Satoh, T. Seya, T. Nakamura, K. Toh, H. Yamagishi, K. Soyama, D. Yamazaki, R. Maruyama, T. Oku, T. Ino, H. Kira, H. Hayashida, K. Sakai, S. Itoh, K. Suzuya, W. Kambara, R. Kajimoto, K. Nakajima, K. Shibata, M. Nakamura, T. Otomo, T. Nakatani, Y. Inamura, J. Suzuki, T. Ito, N. Okazaki, K. Moriyama, K. Aizawa, S. Ohira-Kawamura, and M. Watanabe: Quantum Beam Sci. 1 (2017). https://doi.org/10.3390/ qubs 1020010 
3 H. Tanaka, Y. Sakurai, T. Takata, T. Watanabe, S. Kawabata, M. Suzuki, S.-I. Masunaga, K. Taki, K. Akabori, K. Watanabe, and K. Ono: Rev. Sci. Instrum. 88 (2017). https://doi.org/10.1063/1.4982036

4 K. Watanabe, Y. Kawabata, A. Yamazaki, A. Uritani, T. Iguchi, K. Fukuda, and T. Yanagida: Nucl. Instrum. Methods Phys. Res., Sect. A 802 (2015) 1. https://doi.org/10.1016/j.nima.2015.08.056

5 A. Ishikawa, A. Yamazaki, K. Watanabe, S. Yoshihashi, A. Uritani, K. Fukuda, A. Koike, R. Ogawara, M. Suda, and T. Hamano: Nucl. Instrum. Methods Phys. Res., Sect. A 954 (2020). https://doi.org/10.1016/ j.nima.2018.11.120

6 T. Yanagida, A. Yamaji, N. Kawaguchi, Y. Fujimoto, K. Fukuda, S. Kurosawa, A. Yamazaki, K. Watanabe, Y. Futami, Y. Yokota, A. Uritani, T. Iguchi, A. Yoshikawa, and M. Nikl: Appl. Phys. Express 4 (2011) 10. https:// doi.org/10.1143/APEX.4.106401

7 N. Kawano, N. Kawaguchi, K. Fukuda, G. Okada, and T. Yanagida: J. Mater. Sci., Mater. Electron. 29 (2018) 8964. https://doi.org/10.1007/s10854-018-8918-2

8 C. W. E. van Eijk: Nucl. Instrum. Methods Phys. Res., Sect. A 38 (2004) 337. https://doi.org/10.1016/ j.radmeas.2004.02.004

9 T. Matsumoto, H. Harano, A. Masuda, J. Nishiyama, Y. Sakurai, and A. Uritani: Radiat. Prot. Dosim. 146 (2011) 92. https://doi.org/10.1093/rpd/ncr119 